\title{
SOME CONTRIBUTIONS TO THE THEORY OF RINGS OF OPERATORS. II
}

\author{
BY \\ ERNEST L. GRIFFIN, JR.
}

Preface. In [3], the first paper in this series, we extended various results obtained by von Neumann in $[6 ; 7]$ to general substantial rings. Now, in this paper, we are able to extend them still further-to arbitrary rings of operators.

The plan of this paper is similar to that of [3]. First, we extend the notion of coupling operator to general rings; then, using the crucial Radon-Nikodym Theorem of Dye proved in [2], we show that the coupling operator is an important invariant governing the spacial isomorphism of rings. Finally, these results are applied to questions of topology in rings; the main result in this direction being the fact that the strongest and the $\sigma$-weak topologies are purely algebraic for arbitrary rings. Another result is the fact that the notion of subring is purely algebraic.

The notation used in this paper is essentially the same as that of [3], which in turn is essentially that of Murray and von Neumann in $[5 ; 6 ; 7]$. However, we wish to emphasize the fact that here the symbol $\prec$ between projections $E$ and $F(E \prec F)$ in a ring will denote the fact that $E$ is equivalent to a subprojection of $F$ and will denote a proper ordering only when specifically stated.

In preparing this paper we have received much encouragement and assistance from Professors I. E. Segal, I. Kaplansky, and R. Kadison, which we gratefully acknowledge.

Note: Misonou, in [4], has proved a special case of Theorem 2 of this paper.

1. The coupling operator for rings of type III. We begin by introducing some definitions and a few general lemmas. The first lemma is a useful form of Zorn's Lemma.

Definition 1.1. Let $\subseteq$ be the set of projections possessing a property of projections in a commutative ring $M$. We shall call the property hereditary if the following conditions are satisfied: 1 . For every collection of orthogonal projections $\left(P_{\alpha} \mid \alpha \in \mathfrak{A}\right) \subseteq \subseteq \subseteq$, we have $\sum_{\alpha \in \mathfrak{A}} P_{\alpha}=P \in \mathfrak{S}$ also. 2. If $0 \neq P \in \mathfrak{S}$ and $Q$ is a projection satisfying $0 \neq Q \leqq P$, then $Q \in \mathfrak{S}$.

Lemma 1.1. A set $\mathfrak{S}$ corresponding to some hereditary property contains a maximal projection. Further, if each nonzero projection in the ring contains a nonzero member of $\mathfrak{S}$, then the identity operator I lies in $\mathfrak{S}$.

Presented to the Society, April 26,1952, under the title On some properties of rings of type III; received by the editors August 10, 1954. 
Proof. Using Zorn's Lemma, we select a maximal collection of nonzero, orthogonal projections $\left(P_{\alpha} \mid \alpha \in \mathfrak{A}\right) \subseteq \subseteq$ and let $P=\sum_{\alpha \in \mathfrak{x}} P_{\alpha}$. If $Q \in \subseteq$ then it is clear from the above definition that the projection $Q P^{\perp}$ is either 0 or a member of $\mathbb{S}$. Thus if this projection is not zero then the maximality of our collection is violated, and $P$ is easily seen to be our maximal element of $\subseteq$.

Now, assume that each nonzero projection in the ring contains a nonzero element of $\subseteq$. But then, if $P^{\perp} \neq 0$, we could find a projection $Q$ in $\subseteq$ such that $0 \neq Q \leqq P^{\perp}$; and again the maximality of $P$ is contradicted. Thus $P$ must be $I$ and the proof of this lemma is complete.

Definition 1.2. A projection $E$ in a ring of operators $M$ on a Hilbert space $H$ is said to be cyclic relative to $M$ if there exists a vector $x \in H$ such that $\left[M^{\prime} x\right]=E H$. If $n$ is an infinite cardinal and $P$ is a nonzero projection in the center of a ring $M$, then $P$ is said to have uniform dimension $n$ (abbreviated u.d. $n$ ) if there exists a family $\left(E_{\alpha} \mid \alpha \in \mathfrak{P}\right)$ of orthogonal, equivalent, cyclic projections in $M$ such that $P=\sum_{\alpha \in \mathfrak{R}} E_{\alpha}$. A central projection $P$ is said to have dimension $n$ (abbreviated d. $n$ ) if there exists a family $\left(P_{\alpha} \mid \alpha \in \mathfrak{A}\right)$ of orthogonal, central projections in $M$ such that each $P_{\alpha}$ has u.d. $n$ and $P=\sum_{\alpha \in \mathfrak{Q}} P_{\alpha}$.

LEMMA 1.2. Let $\left(E_{\alpha} \mid \alpha \in \mathfrak{A}\right),\left(F_{\mathrm{e}} \mid \epsilon \in \mathbb{E}\right)$ be infinite families of orthogonal, cyclic projections in a ring $M$ on a Hilbert space $H$. If $\sum_{\alpha \in \mathfrak{A}} E_{\alpha}=\sum_{\epsilon \in \mathbb{E}} F_{\epsilon}=I$, then the cardinal of the set $\mathfrak{A}$ equals that of the set $\mathfrak{E}$.

Proof. Let $n, n^{\prime}$ be the cardinals of $\mathfrak{A}$, $\mathbb{E}$ respectively, and set $\Im_{\alpha}=\left(\epsilon \in \mathbb{F} \mid E_{\alpha} F_{\epsilon}\right.$ $\not 00)$. For any $\epsilon \in\left(\xi ;\right.$ if $E_{\alpha} F_{\epsilon}=0$ for all $\alpha \in \mathfrak{A}$, then $\sum_{\alpha \in \mathfrak{A}} E_{\alpha} F_{\epsilon}=F_{\epsilon}=0$. This contradiction proves that for each $\epsilon \in \mathbb{E}$ there exists an $\alpha(\epsilon) \in \mathfrak{A}$ such that $E_{\alpha(\epsilon)} F_{\epsilon} \neq 0$, but then $\epsilon \in \Im_{\alpha(\epsilon)}$, yielding finally

$$
\mathfrak{F}=\bigcup_{\alpha \in \mathfrak{Q}} \Im_{\alpha} \text {. }
$$

Since the $E_{\alpha}$ are cyclic, we can find vectors $\left(x_{\alpha} \mid \alpha \in \mathfrak{A}\right) \subseteq H$ such that $\left[M^{\prime} x_{\alpha}\right]=E_{\alpha} H$ for all $\alpha \in \mathfrak{A}$. Now if $0=\left(F_{\epsilon} x_{\alpha}, x_{\alpha}\right)=\left\|F_{\epsilon} x_{\alpha}\right\|^{2}$, then $F_{\epsilon} x_{\alpha}=0$, which with $F_{\epsilon} M^{\prime} x_{\alpha}=M^{\prime} F_{\epsilon} x_{\alpha}=0$ finally implies $0=F_{\epsilon} E_{\alpha}=\left(E_{\alpha} F_{\epsilon}\right)^{*}$. Thus $\varsigma_{\alpha} \subseteq\left(\epsilon \in \mathbb{E} \mid\left(F_{\epsilon} x_{\alpha}, x_{\alpha}\right) \neq 0\right)$, and the argument of Lemma 1.1.2, page 472 of [3] shows that the sets $\mathfrak{S}_{\alpha}$ are each countable.

It is now clear that $n^{\prime}=\left[\right.$ cardinal of $\left.\left(U_{\alpha \in \mathfrak{n}} \subseteq_{\alpha}\right)\right] \leqq \boldsymbol{\aleph}_{0} \cdot n=n$. By symmetry, $n \leqq n^{\prime}$, with finally $n=n^{\prime}$. This completes the proof.

LEMMA 1.3. Let $M$ be a purely infinite ring operating on a Hilbert space $H$. There exists an infinite cardinal $n$ and a central projection $P$ such that $P$ has u.d.n.

Proof. Since the ring is purely infinite, we can find a family $\left(E_{\alpha} \mid \alpha \in \mathfrak{A}\right)$ of infinitely many nonzero, orthogonal, equivalent, cyclic projections in $M$. By Zorn's Lemma we can assume that the family is maximal and form 
$E=\sum_{\alpha \in \mathfrak{A}} E_{\alpha}$. Comparing $E_{\alpha}$ with $E^{\perp}$, we notice that $E_{\alpha}<E^{\perp}$ is false by the maximality of our family; and thus by Theorem 6, page 222 of [1], there exists a projection $P$ in the center of $M$ such that $P\left(E^{\perp}\right) \prec P E_{\alpha}$ (proper).

We now have $P=\sum_{\alpha \in \mathfrak{A}} P E_{\alpha}+P\left(E^{\perp}\right)$, and the projections $P E_{\alpha}$ form an orthogonal family of equivalent, cyclic projections. Let $\alpha_{0}$ be a fixed element of $\mathfrak{A}$ and consider the fact that $\sum_{\alpha \in \mathfrak{A}_{-\alpha_{0}}} P E_{\alpha} \sim \sum_{\alpha \in \mathfrak{A}} P E_{\alpha}$ as each of the index sets has the same infinite cardinality $n$. But this leads to

$$
\sum_{\alpha \in \mathfrak{q}} P E_{\alpha}>\sum_{\alpha \in \mathfrak{q}-\alpha_{0}} P E_{\alpha}+P\left(E^{\perp}\right) \sim \sum_{\alpha \in \mathfrak{M}} P E_{\alpha}+P\left(E^{\perp}\right)=P
$$

or $P \sim \sum_{\alpha \in \mathfrak{A}} P E_{\alpha}$.

Next, select a partial isometry $V$ in $M$ such that $V^{*} V=P$ and $V V^{*}$ $=\sum_{\alpha \in \mathfrak{A}} P E_{\alpha}$. Then $P=V^{*} V V^{*} V=V^{*}\left(\sum_{\alpha \in \mathfrak{A}} P E_{\alpha}\right) V=\sum_{\alpha \in \mathfrak{A}} P V^{*} E_{\alpha} V$. But now if we let $W=P E_{\alpha} V$, then $W^{*} W=P V^{*} E_{\alpha} V$ and $W W^{*}=P E_{\alpha}$ shows that the family $\left(P V^{*} E_{\alpha} V \mid \alpha \in \mathfrak{A}\right)$ consists of equivalent, cyclic projections, with finally $P$ having u.d.n. This completes the proof of the lemma.

Lemma 1.4. Let $P$ be a projection of dimension $n$ in the center of a ring $M$ on a Hilbert space $H$. $P$ has uniform dimension $n$ if and only if $\left(M \cap M^{\prime}\right)_{P H}$ is countably decomposable.

Proof. There is clearly no loss of generality in assuming that $P=I$. First, we assume that $I$ is of u.d. $n$; then there exists a family $\left(E_{\alpha} \mid \alpha \in \mathfrak{A}\right)$ of orthogonal, equivalent projections in $M$ such that $\sum_{\alpha \in \mathfrak{R}} E_{\alpha}=I$, and there exists a family $\left(x_{\alpha} \mid \alpha \in \mathfrak{A}\right)$ of vectors in $H$ such that $\left[M^{\prime} x_{\alpha}\right]=E_{\alpha} H$ for each $\alpha \in \mathfrak{A}$. Now define the linear functional $\beta$ on $M \cap M^{\prime}$ by $\beta(T)=\left(T x_{\alpha}, x_{\alpha}\right)$ for some $\alpha \in \mathfrak{A}$, and assume that $Q$ is a projection in $M \cap M^{\prime}$ such that $\beta(Q)=0$. But this yields $\left(Q x_{\alpha}, x_{\alpha}\right)=\left\|Q x_{\alpha}\right\|^{2}=0$, or $Q x_{\alpha}=0$. At once we get $Q M^{\prime} x_{\alpha}=M^{\prime} Q x_{\alpha}=0$, and then $Q E_{\alpha}=0$. We next use the equivalence of the $E_{\alpha}$ to get $Q=0$. Now since $\beta(Q)=0$ is seen to imply $Q=0$, we can apply Lemma 1.1.2, page 472 of [3] to get $M \cap M^{\prime}$ countably decomposable.

To complete the proof, now assume that $M \cap M^{\prime}$ is countably decomposable. The identity, by hypothesis, can be split up into a sum of orthogonal, central projections $\left(P_{\epsilon} \mid \epsilon \in \mathbb{E}\right) \subseteq M$ each having u.d.n. Consequently, there exist families $\left[\left(E_{\epsilon, \alpha} \mid \alpha \in \mathfrak{R}\right) \mid \epsilon \in \mathbb{E}\right]$ of orthogonal, equivalent (for the same $\epsilon$ ) projections in $M$ along with vectors $\left(x_{\epsilon}, \alpha \mid \alpha \in \mathfrak{A}, \epsilon \in \mathbb{F}\right)$ such that $\sum_{\alpha \in \mathfrak{X}} E_{\epsilon, \alpha}$ $=P_{\epsilon}$ and $\left[M^{\prime} x_{\epsilon, \alpha}\right]=E_{\epsilon, \alpha} H$. (We can pick the same index set $\mathfrak{A}$ for each $\epsilon$ since each $P_{c}$ has the same uniform dimension.) Further, since the set $\&$ is countable by hypothesis, we can assume that vectors $\left(x_{\alpha} \mid \alpha \in \mathfrak{A}\right)$ exist such that $x_{\alpha}=\sum_{\epsilon \in \mathbb{E}} x_{\epsilon, \alpha}$.

We now let $F_{\alpha}$ be the projection determined by $\left[M^{\prime} x_{\alpha}\right]$ and form $E_{\alpha}$ $=\sum_{\epsilon \in \mathbb{G}} E_{\epsilon, \alpha}$. The equations $E_{\alpha} x_{\alpha}=\sum_{\epsilon \in \mathcal{G}} E_{\alpha} x_{\epsilon, \alpha}=\sum_{\epsilon \in \mathfrak{C}} x_{\epsilon, \alpha}=x_{\alpha}$ show clearly that $E_{\alpha} M^{\prime} x_{\alpha}=M^{\prime} E_{\alpha} x_{\alpha}=M^{\prime} x_{\alpha}$ and that $F_{\alpha} \leqq E_{\alpha}$. But [ $\left.M^{\prime} x_{\alpha}\right]$ $\supseteq\left[M^{\prime} P_{\epsilon} x_{\alpha}\right]=\left[M^{\prime} x_{\epsilon, \alpha}\right]=E_{\epsilon, \alpha} H$ leads to $E_{\epsilon, \alpha} \leqq F_{\alpha}$ and consequently 


$$
E_{\alpha}=\sum_{\epsilon \in \mathfrak{E}} E_{\epsilon, \alpha} \leqq F_{\alpha} .
$$

Thus $E_{\alpha}=F_{\alpha}$, and the $E_{\alpha}$ are seen to form a family of equivalent cyclic projections adding up to $I$ by the computation: $\sum_{\alpha \in \mathfrak{l}} E_{\alpha}=\sum_{\mathfrak{\epsilon} \in \mathbb{E}, \alpha \in \mathfrak{q}} E_{\mathfrak{\epsilon}, \boldsymbol{\alpha}}$ $=\sum_{\epsilon \in \in} P_{\epsilon}=I$. This proves that $I$ has u.d.n.

Lemma 1.5. If $E$ is a projection in a ring $M$ of type III on a Hilbert space $H$, then $E$ is cyclic relative to $M$ if and only if $M_{E H}$ is countably decomposable.

Proof. Again it is clear that there is no loss of generality in assuming that $E$ is the identity $I$. We first assume that $\left[M^{\prime} x\right]$ equals $H$ and consider the linear functional $\beta$ defined on $M$ by the equation $\beta(T)=(T x, x)$ for $T$ in $M$. Just as in the case of the $\beta$ in the proof of Lemma 1.4 above, $\beta(Q)=0$ for a projection $Q$ in $M$ implies $Q x=0$ and hence $Q H=Q\left[M^{\prime} x\right]=\left[Q M^{\prime} x\right]=\left[M^{\prime} Q x\right]$ $=0$. Thus the countable decomposability of $M$ follows from Lemma 1.1.2, page 472 of [3].

Now assume that $M$ is countably decomposable. It is clear from Lemma 1.3 that there exist central projections of dimension $\boldsymbol{N}_{0}$ in $M$; and, since the property of having a given dimension is obviously hereditary (Definition 1.1 ), we can apply Lemma 1.1 to show that $I$ has dimension $\boldsymbol{\aleph}_{0}$. Further, it follows from Lemma 1.4 that the identity operator $I$ has uniform dimension $\aleph_{0}$.

To complete the proof of this lemma, now select a family $\left(E_{\alpha} \mid \alpha \in \mathfrak{A}\right)$ of orthogonal, equivalent, cyclic projections in $M$ such that $\sum_{\alpha \in \mathfrak{A}} E_{\alpha}=I$, using the fact that $I$ has u.d. $\boldsymbol{\aleph}_{0}$. But since $M$ is of type III, each $E_{\alpha}$ is infinite; and if $E$ is one of the $E_{\alpha}$, then the ring $M_{E H}$ is countably decomposable and of type III. Thus, by the argument of the previous paragraph, $E$ has u.d. $\boldsymbol{\aleph}_{0}$ relative to $M_{E H}$; and hence $E$ can be written as a sum of countably many equivalent, orthogonal projections. We now use $E \sim E_{\alpha}, \alpha \in \mathfrak{A}$, to obtain equivalent, orthogonal projections $\left(E_{\alpha, \epsilon} \mid \alpha \in \mathfrak{A}, \epsilon \in \mathfrak{E}\right)$ such that $\sum_{\epsilon \in \in} E_{\alpha, \epsilon}$ $=E_{\alpha}$ for all $\alpha \in \mathfrak{A}$; and we notice that $\sum_{\alpha \in \mathfrak{A}, \epsilon \in \mathfrak{E}} E_{\alpha, \mathfrak{\epsilon}}=\sum_{\alpha=\mathfrak{A}} E_{\alpha}=I$. Since the sets $\mathfrak{A}$ and $\mathbb{F}$ are both countable, we see that $I$ and each of the $E_{\alpha}$ are sums of countably many of the $E_{\alpha, \epsilon}$ and are therefore equivalent. Finally, since the $E_{\alpha}$ are cyclic, $I$ itself must be also.

We are now ready to prove

Theorem 1. Let $M$ be a ring of type III on the Hilbert space $H$, and let $\mathfrak{N}$ be the collection of infinite cardinals which are less than or equal to the cardinal of $H$. For each $n$ in $\mathfrak{N}$ there exists a projection $P_{n}$ in the center of $M$ such that either $P_{n}=0$ or $P_{n}$ is dimension $n$ and such that $\sum_{n \in \mathfrak{N}} P_{n}=I$. Further, this decomposition is purely algebraic; in fact, the properties of being of u.d.n and of being cyclic are purely algebraic.

Proof. The purely algebraic nature of cyclic projections in rings of type III clearly follows from Lemma 1.5 plus the fact that countable decomposability 
is purely algebraic. Hence if a central projection is a sum of a family of $n$ equivalent, cyclic, orthogonal projections, its image under a ${ }^{*}$-isomorphism will be also. This proves that the property of being u.d. $n$ is purely algebraic, and it is clear that being of dimension $n$ is too.

Further, it is clear that the property of being of dimension $n$ is hereditary with respect to the center of $M$; and thus for each $n \in \mathfrak{R}$ which is the dimension of some central projection in $M$ we can use Lemma 1.1 to get a maximal projection $P_{n}$ with d. $n$. If there is no central projection of dimension $n$ for some $n \in \mathfrak{R}$ then we define $P_{n}=0$.

Now let $P=\sum_{n \in \mathfrak{R}} P_{n}$. If $P \neq I$, apply Lemma 1.3 to the ring $M(I-P)$ to find a nonzero central projection $Q \leqq I-P$ which has u.d. $n$ for some $n \in \mathfrak{N}$. But by our choice of $P_{n}$ we get $Q=Q P_{n}=Q P P_{n}=0$, which proves that $P$ must have been equal to $I$; and the proof of our theorem is complete.

With Theorem 1 out of the way we are now able to define a "coupling operator." The aptness of this definition will be more evident after the proof of Theorem 2 in the next section. But first we have

Definition 1.3. Let $M$ be a ring of type III on the Hilbert space $H$, and let $\left(P_{n} \mid n \in \mathfrak{R}\right),\left(P_{n}^{\prime} \mid n \in \mathfrak{R}\right)$ be decompositions of the identity in the rings $M, M^{\prime}$ respectively; the decompositions being those mentioned in the statement of Theorem 1 above. We now introduce the formal operators $\left(n / n^{\prime}\right) P_{n} P_{n^{\prime}}^{\prime}$ and the formal sum $C=\sum_{n, n^{\prime} \in \mathfrak{N}}\left(n / n^{\prime}\right) P_{n} P_{n^{\prime}}^{\prime}$. The formal operator $C$ will be called the coupling operator for the pair $M, M^{\prime}$.

2. *-isomorphisms of rings of type III. This section will be devoted to the proof of the type III analog of Theorem 9, page 497 of [3] which yields a condition for the spacial isomorphism of certain *-isomorphic rings of operators. We first prove

Lemma 2.1. Let $M$ be a ring of type III on a Hilbert space H. If $M$ is countably decomposable, then every projection in $M$ is equivalent to some central projection.

Proof. Let $E$ be an arbitrary nonzero projection in $M$. Since $M_{E H}$ is of type III and is countably decomposable, it is clear from Theorem 1 that $E$ is of dimension $\boldsymbol{\aleph}_{0}$; in fact, Lemma 1.4 shows that $E$ is of uniform dimension. Hence $E$ can be expressed as the sum of the collection $\left(F_{\alpha} \mid \alpha \in \mathfrak{A}\right)$ of countably many orthogonal, equivalent, cyclic projections in $M$; and we, by Zorn's Lemma, extend this collection to be maximal in $M$ with respect to being orthogonal and equivalent, obtaining the family $\left(F_{\alpha} \mid \alpha \in \mathfrak{A}^{\prime} \supseteq \mathfrak{A}\right)$. Let $F=\sum_{\alpha \in \mathfrak{r},} F_{\alpha}$.

We now proceed as in the proof of Lemma 1.3, comparing $F_{\alpha}$ with $F^{\perp}$ and obtaining a projection $P$ in the center of $M$ such that $P\left(F^{\perp}\right) \prec P F_{\alpha}$ (proper) and finally proving that $0 \neq P \sim \sum_{\alpha \in \mathfrak{R}}, P F_{\alpha}$. Then $P E=\sum_{\alpha \in \mathfrak{X}} P F_{\alpha}$; and since the index sets $\mathfrak{A}$ and $\mathfrak{H}^{\prime}$ are both countable, we get the result $P E \sim P$. 
We next observe that the property of central projections $P$ to satisfy $P E \sim P$ is hereditary and use Lemma 1.1 to obtain a maximal projection $Q$ with that property. However if $Q^{\perp} E \neq 0$, we could apply the argument of the preceding two paragraphs to get a central projection $P$ such that $0 \neq P \leqq Q^{\perp}$ and $P E \sim P$, thus contradicting the maximality of $Q$. Hence $Q^{\perp} E=0$, and $Q \sim Q E=Q E+Q^{\perp} E=E$. This completes the proof of the lemma.

TheOREM 2. Let $M, M^{\prime}, \tilde{M}, \tilde{M}^{\prime}$, be rings of type III on Hilbert spaces $H$, $\tilde{H}$ respectively. If $C$ and $\tilde{C}$ are the respective coupling operators and if $\phi$ is a *-isomorphism of $M$ onto $\tilde{M}$ taking $C$ into $\tilde{C}$, then there exists a linear isometry $W$ mapping $H$ onto $\tilde{H}$ such that $\phi(A)=W A W^{-1}$ for $A$ in $M$.

Proof. Let $\left(P_{n} \mid n \in \mathfrak{N}\right),\left(P_{n}^{\prime} \mid n \in \mathfrak{N}\right)$ be the decompositions of the identity in $M$ mentioned in the definition of the coupling operator. By our hypothesis of $\phi(C)=\tilde{C}$, it is clear that $\left(\phi\left(P_{n}\right) \mid n \in \mathfrak{N}\right)$ and $\left.\left(\phi\left(P_{n}^{\prime}\right) \mid n \in \mathfrak{N}\right)\right)$ are the corresponding decompositions of the identity in $\tilde{M}$. We first observe that if we can find isometries on the manifolds $\left(P_{n} P_{n^{\prime}}^{\prime} H \mid n, n^{\prime} \in \mathfrak{N}\right)$ implementing $\phi$, that we can find one in the large by merely piecing together these partial isometries.

Therefore we need only consider cases in which the identity has dimensions $n, n^{\prime}$ with respect to the rings $M, M^{\prime}$ respectively. Next we notice that we can further decompose $I$ into central projections $\left(P_{\alpha} \mid \alpha \in \mathfrak{A}\right)$ which have uniform dimension $n$. But by Lemma 1.4 this implies that each of the rings $\left(M \cap M^{\prime}\right) P_{\alpha}$ is countably decomposable; and hence that since the $P_{\alpha}$ are of dimension $n^{\prime}$ with respect to $M^{\prime}$, that they are of uniform dimension with respect to $M^{\prime}$ also. This finally reduces us to the consideration of cases in which $I$ has uniform dimensions $n, n^{\prime}$ with respect to $M, M^{\prime}$ respectively. $I$ will now be u.d. $n$ with respect to $\tilde{M}$ by Theorem 1 and u.d. $n^{\prime}$ with respect to $\tilde{M}^{\prime}$ by Lemma 1.4 . We now proceed to the proof of:

CASE 1. $M, M^{\prime}, \tilde{M}, \tilde{M}^{\prime}$ all countably decomposable. Choose a nonzero vector $\tilde{x} \in \tilde{H}$ and consider the functional $(\phi(A) \tilde{x}, \tilde{x})$ defined for $A \in M$. This functional is obviously positive linear and countably additive and thus clearly satisfies the hypotheses of Theorem 1, page 247 of [2]. Hence there exists a nonzero projection $E$ in $M$ and a vector $y$ in $E H$ such that for each operator $A$ in $M,(A y, y)=\left(A E y, E_{y}\right)=(E A E y, y)=(\phi(E A E) \tilde{x}, \bar{x})=(\phi(E) \phi(A) \phi(E) \bar{x}, \tilde{x})$ $=(\phi(A) \phi(E) \tilde{x}, \phi(E) \tilde{x})$. If we let $\phi(E) \tilde{x}=\tilde{y}$, then by the above computation, $\|A y\|^{2}=\left(A^{*} A y, \quad y\right)=\left(\phi\left(A^{*} A\right) \tilde{y}, \quad \tilde{y}\right)=\left(\phi\left(A^{*}\right) \phi(A) \tilde{y}, \quad \tilde{y}\right)=\left(\phi(A)^{*} \phi(A) \tilde{y}, \quad \tilde{y}\right)$ $=\|\phi(A) \tilde{y}\|^{2}$ for all operators $A$ in $M$.

The above computation shows that we can define a mapping $W_{0}$ of $[M y]$ onto $[\tilde{M} \tilde{y}]$ by $W_{0} A y=\phi(A) \tilde{y}$ and extending to the closures in the standard manner. $W_{0}$ is clearly a linear isometry; and if we let $E^{\prime}=P_{[M y]}, \tilde{E}^{\prime}=P_{[\tilde{M} \tilde{y}]}$, $A \in M$, and $\tilde{B} \in \tilde{M}$ then $W_{0} A E^{\prime} W_{0}^{-1} \tilde{B} \tilde{y}=W_{0} A E^{\prime} \phi^{-1}(\widetilde{B}) y=W_{0} A \phi^{-1}(\widetilde{B}) y$ $=\phi(A) \tilde{B} \tilde{y}=\phi(A) \tilde{E}^{\prime} \tilde{B} \tilde{y}$. This proves that $W_{0} A E^{\prime} W_{0}^{-1}=\phi(A) \tilde{E}^{\prime}$ by density of $\tilde{M} \tilde{y}$ in $\tilde{E}^{\prime} \tilde{H}$. 
We next apply Lemma 2.1 to $E^{\prime}$ and $E^{\prime}$ and obtain central projections $P$ and $\widetilde{P}$ such that $E^{\prime} \sim P$ and $\tilde{E}^{\prime} \sim \widetilde{P}$. Thus there exist partial isometries $V^{\prime}, \tilde{V}^{\prime}$ such that $V^{*} V^{\prime}=E^{\prime}, V^{\prime} V^{*}=P, \tilde{V}^{*} \tilde{V}^{\prime}=\tilde{E}^{\prime}$, and $\tilde{V}^{\prime} \tilde{V}^{\prime *}=\tilde{P}$. Further, we define $W_{1}=\tilde{V}^{\prime} W_{0} V^{\prime *}$; notice that $W_{1}$ is a linear isometry mapping $P H$ onto $\widetilde{P} \tilde{H}$; and, for $A \in P M$, consider the operator $W_{1} A W_{1}^{-1}$ on $\widetilde{P} \widetilde{H}$. We see readily that

$$
\begin{aligned}
W_{1} A W_{1}^{-1}=\tilde{V}^{\prime} W_{0} V^{\prime *} A V^{\prime} W_{0}^{-1} \tilde{V}^{\prime *} & =\tilde{V}^{\prime} W_{0} V^{\prime *} V^{\prime} A W_{0}^{-1} \tilde{V}^{\prime *} \\
& =\tilde{V}^{\prime} W_{0} E^{\prime} A W_{0}^{-1} \tilde{V}^{\prime *} \quad\left(\text { by } V^{\prime *} V^{\prime}=E^{\prime}\right) \\
& =\tilde{V}^{\prime}\left[\phi(A) \tilde{E}^{\prime}\right] \tilde{V}^{\prime *} \quad\left(W_{0} \text { implements } \phi \text { on } E^{\prime} H\right) \\
& =\phi(A) \tilde{V}^{\prime} \tilde{E}^{\prime} \tilde{V}^{\prime *}=\phi(A) \tilde{V}^{\prime} \tilde{V}^{\prime *} \tilde{V}^{\prime} \tilde{V}^{\prime *} \\
& =\phi(A) \tilde{V}^{\prime} \tilde{V}^{\prime *} \quad\left(\text { by } \tilde{V}^{\prime} \tilde{V}^{\prime *} \tilde{V}^{\prime}=\tilde{V}^{\prime}\right) \\
& =\phi(A) \tilde{P} .
\end{aligned}
$$

Now, if $A=P$, we get $W_{1} W_{1}^{-1}=\widetilde{P}=\phi(P) \tilde{P}$, or $\tilde{P} \leqq \phi(P)$. By a similar argument we get $\phi(P) \leqq \tilde{P}$, so $\phi(P)=\tilde{P}$. Therefore, by the above computation, for $A \in P M$,

$$
W_{1} A W_{1}^{-1}=\phi(A) \widetilde{P}=\phi(A) \phi(P)=\phi(A P)=\phi(A)
$$

and we have been able to implement $\phi$ on $P H$.

We have now proved that every ${ }^{*}$-isomorphism which satisfies the hypotheses of Case 1 can be implemented at least on a nontrivial manifold belonging to the center of our ring. But the property of having an implementation is obviously hereditary and thus we can use Lemma 1.1 to show that our *-isomorphism $\phi$ can be implemented on $H$, since $I$ is our maximal element having that property. This completes the proof of Case 1 .

CASE 2. $M, \tilde{M}$ countably decomposable and $M^{\prime}, \tilde{M}^{\prime}$ with u.d.n'. Using an index set $\mathfrak{A}$ of cardinality $n^{\prime}$ we select families of projections $\left(E_{\alpha}^{\prime} \mid a \in \mathfrak{A}\right)$, $\left(\tilde{E}_{\alpha}^{\prime} \mid \alpha \in \mathfrak{A}\right)$ as in Definition 1.2 , since $I$ and $I$ have u.d. $n^{\prime}$ relative to $M^{\prime}, \tilde{M}^{\prime}$ respectively. If we let $1 \in \mathfrak{A}$, we can find partial isometries $\left(V_{\alpha}^{\prime} \mid \alpha \in \mathfrak{A}\right)$ mapping $E_{1}^{\prime} H$ isometrically onto the manifolds $E_{\alpha}^{\prime} H$. If we do the same in $\tilde{M}$, we end up finally with families of partial isometries $\left(V_{\alpha}^{\prime} \mid \alpha \in \mathfrak{A}\right),\left(\tilde{V}_{\alpha}^{\prime} \mid \alpha \in \mathfrak{A}\right)$ such that for all $\alpha \in \mathfrak{A}, V_{\alpha}^{\prime} V_{\alpha}^{\prime}=E_{1}^{\prime}, \widetilde{V}_{\alpha}^{\prime *} \widetilde{V}_{\alpha}^{\prime}=\widetilde{E}_{1}^{\prime}, V_{\alpha}^{\prime} V_{\alpha}^{\prime *}=E_{\alpha}^{\prime}$, and $\tilde{V}_{\alpha}^{\prime} \tilde{V}_{\alpha}^{\prime *}=\tilde{E}_{\alpha}^{\prime}$.

We now consider the mapping $B \rightarrow B E_{1}^{\prime}$ of $M$ into $M_{E_{1}^{\prime} H}$. If $B E_{1}^{\prime}=0$ for $B \in M$, then $0=B V_{\alpha}^{\prime *} V_{\alpha}^{\prime}=V_{\alpha}^{\prime}\left(B V_{\alpha}^{\prime}{ }^{*} V_{\alpha}^{\prime}\right)=B\left(V_{\alpha}^{\prime} V_{\alpha}^{\prime *} V_{\alpha}^{\prime}\right)=B V_{\alpha}^{\prime}$ $=\left(B V_{\alpha}^{\prime}\right) V_{\alpha}^{\prime *}=B E_{\alpha}^{\prime}$ for all $\alpha \in \mathfrak{A}$; thus $B E_{1}^{\prime}=0$ implies $0=\sum_{\alpha \in \mathfrak{X}} E_{\alpha}^{\prime} B$ $=I B=B$. Therefore our mapping is a ${ }^{*}$-isomorphism of $M$ onto $M_{E_{1}{ }^{\prime} H}$, and a similar argument shows that the mapping $\widetilde{B} \rightarrow \widetilde{B} \tilde{E}_{1}^{\prime}$ is a *-isomorphism of $\tilde{M}$ onto $\tilde{M}_{\tilde{E}^{\prime}, \tilde{H}}$. Also, the mapping $B E_{1}^{\prime} \rightarrow \phi(B) \tilde{E}_{1}^{\prime}$ is a ${ }^{*}$-isomorphism of $M_{E^{\prime} H}$ onto $\widetilde{M}_{\widetilde{E}_{1}^{\prime} \tilde{B}}$ since it can be factored into the ${ }^{*}$-isomorphisms: $B E_{1}^{\prime} \rightarrow B$ $\rightarrow \phi(B) \rightarrow \phi(B) \tilde{E}_{1}^{\prime}$. 
Next, if $x$ is the vector which generates the cyclic projection $E_{1}^{\prime}$, then the computation $\left[M_{E^{\prime}{ }_{1} H} x\right]=\left[M E_{1}^{\prime} x\right]=[M x]=E_{1}^{\prime} H$ plus Lemma 1.5 shows that the ring $M_{E^{\prime} H}^{\prime}$ is countably decomposable. Since a similar argument shows that $\widetilde{M}^{\prime} \widetilde{E} \widetilde{H}^{\prime}$ is also countably decomposable, we arrive at the conclusion that all the rings $M_{E^{\prime}{ }_{1} H}, M_{E^{\prime}{ }_{1} H}^{\prime}, \widetilde{M}_{\widetilde{E}^{\prime}{ }_{1} \tilde{H}}, \widetilde{M}^{\prime} \widetilde{E}_{{ }^{\prime}} \widetilde{H}$ are countably decomposable and that

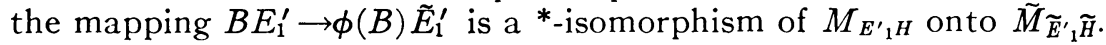

With the results of the preceding paragraph, we can apply Case 1 of the present theorem to obtain a linear isometry $W_{0}$ mapping $E_{1}^{\prime} H$ onto $\tilde{E}^{\prime} \tilde{H}$, implementing the *-isomorphism induced by $\phi$. Further, it is clear from our previous argument that the mapping $\tilde{V}_{\alpha}^{\prime} W_{0} V_{\alpha}^{\prime *}$ takes $E_{\alpha}^{\prime} H$ into $\tilde{E}_{\alpha}^{\prime} \widetilde{H}$, and hence the linear isometry $W=\sum_{\alpha \in \mathfrak{A}} \tilde{V}_{\alpha}^{\prime} W_{0} V_{\alpha}^{\prime *}$ maps $H$ onto $\widetilde{H}$.

Now, for $\beta$ arbitrary in $\mathfrak{A}, \quad W E_{\beta}^{\prime} W^{-1}=\sum_{\alpha \in \mathfrak{A}} \quad \tilde{V}_{\alpha}^{\prime} W_{0} V_{\alpha}^{\prime *} E_{\beta}^{\prime} W^{-1}$ $=\tilde{V}_{\beta}^{\prime} W_{0} V_{\beta}^{\prime *} W^{-1}=\tilde{V}_{\beta}^{\prime} W_{0} V_{\beta}^{\prime *}\left(\sum_{\alpha \in \mathfrak{I}} V_{\alpha}^{\prime} W_{0}^{-1} \tilde{V}^{\prime *}\right)=\tilde{V}_{\beta}^{\prime} W_{0} V_{\beta}^{\prime *} V_{\beta}^{\prime} W_{0}^{-1} \tilde{V}_{\beta}^{\prime *}=\tilde{V}_{\beta}^{\prime}$ $W_{0} E_{1}^{\prime} W_{0}^{-1} \tilde{V}_{\beta}^{\prime *}=\tilde{V}_{\beta}^{\prime} \tilde{E}_{1}^{\prime} \tilde{V}_{\beta}^{\prime *}=\tilde{E}_{\beta}^{\prime}$, by the properties of our partial isometries $\left(V_{\alpha}^{\prime}, \tilde{V}_{\alpha}^{\prime} \mid \alpha \in \mathfrak{A}\right)$. Also, for arbitrary $B \in M$ and finite subset $\mathfrak{A}_{0}$ of $\mathfrak{A}$, we get a similar computation:

$$
\begin{aligned}
\left(\sum_{\alpha \in \mathfrak{I}_{0}} \tilde{V}_{\alpha}^{\prime} W_{0} V_{\alpha}^{\prime *}\right) & B\left(\sum_{\alpha \in \mathfrak{R}_{0}} V_{\alpha}^{\prime} W_{0}^{-1} \tilde{V}_{\alpha}^{\prime *}\right) \\
& =\sum_{\alpha, \beta \in \mathfrak{I}_{0}} \tilde{V}_{\alpha}^{\prime} W_{0} V_{\alpha}^{\prime *} B V_{\beta}^{\prime} W_{0}^{-1} \tilde{V}_{\beta}^{\prime *} \\
& =\sum_{\alpha, \beta \in \mathfrak{I}_{0}} \tilde{V}_{\alpha}^{\prime} W_{0}\left(B V_{\alpha}^{\prime *} V_{\beta}^{\prime}\right) W_{0}^{-1} \tilde{V}_{\beta}^{\prime *} \quad\left(B \text { commutes with } V_{\alpha}^{\prime *}\right) \\
& \left.=\sum_{\alpha \in \mathfrak{I}_{0}} \tilde{V}_{\alpha}^{\prime} W_{0}\left(B E_{1}^{\prime}\right) W_{0}^{-1} \tilde{V}_{\alpha}^{\prime *} \quad \text { (since } V_{\alpha}^{\prime *} V_{\beta}^{\prime}=0 \text { for } \alpha \neq \beta\right) \\
& =\sum_{\alpha \in \mathfrak{I}_{0}} \tilde{V}_{\alpha}^{\prime} \phi(B) \tilde{E}_{1}^{\prime} \tilde{V}_{\alpha}^{\prime *}=\sum_{\alpha \in \mathfrak{I}_{0}} \phi(B) \tilde{V}_{\alpha}^{\prime} \tilde{E}_{1}^{\prime} \tilde{V}_{\alpha}^{\prime *} \\
& =\phi(B) \sum_{\alpha \in \mathfrak{I}_{0}} \tilde{E}_{\alpha}^{\prime} .
\end{aligned}
$$

Since our set $\mathfrak{A}_{0}$ was arbitrary in $\mathfrak{A}$, an obvious continuity argument proves:

$$
W B W^{-1}=\phi(B) \text {. }
$$

This clearly completes the proof of Case 2 .

CASE 3. I, I of u.d.n with respect to $M, \tilde{M}$ respectively and u.d.n' with respect to $M^{\prime}, \tilde{M}^{\prime}$ respectively. Since $I$ has u.d.n relative to $M$, there exists a family $\left(E_{\alpha} \mid \alpha \in \mathfrak{A}\right)$ of $n$ orthogonal, cyclic, equivalent projections in $M$ such that $\sum_{\alpha \in \mathfrak{I}} E_{\alpha}=I$. If we let $\phi\left(E_{\alpha}\right)=\tilde{E}_{\alpha}$, the family $\left(\tilde{E}_{\alpha} \mid \alpha \in \mathfrak{A}\right)$ is a family of projections in $\tilde{M}$ with properties similar to those of the $E_{\alpha}$, by Theorem 1. For $1 \in \mathfrak{A}$, there exist partial isometries $\left(V_{\alpha} \mid \alpha \in \mathfrak{U}\right)$ in $M$ such that $V_{\alpha} V_{\alpha}^{*}=E_{\alpha}$. $V_{\alpha}^{*} V_{\alpha}=E_{1}$.

Next, we notice that $\phi$ induces a ${ }^{*}$-isomorphism of $M_{E_{1} H}$ onto $\widetilde{M}_{\widetilde{E}_{1} \widetilde{H}}$; and, by the argument of the second paragraph of our proof of Case 2 of the 
present theorem, we find that the rings $M^{\prime}, \tilde{M}^{\prime}$ are *-isomorphic to the rings $M_{E_{1} H}^{\prime}, \widetilde{M}^{\prime} \widetilde{E}_{1} \widetilde{H}$ respectively. Further, if $x$ is the vector which generates the cyclic projection $E_{1}$, then $\left[M_{E_{1} H}^{\prime} x\right]=\left[M^{\prime} E_{1} x\right]=\left[M^{\prime} x\right]$ and Lemma 1.5 prove that the ring $M_{E_{1} H}$ is countably decomposable.

Thus we have proved that rings $M_{E_{1} H}, M_{E_{1} H}^{\prime}, \widetilde{M}_{\tilde{E}_{1} \tilde{H}}$, and $\tilde{M}^{\prime} \widetilde{E}_{1} \tilde{H}$ satisfy the hypotheses of our Case 2 above, since the first and third of the rings are countably decomposable and the second and fourth have identity operators possessing u.d. $n^{\prime}$ (by Theorem 1). This yields a linear isometry $W_{0}$ mapping $E_{1} H$ onto $\widetilde{E}_{1} \tilde{H}$ with the property: $W_{0} B W_{0}^{-1}=\phi(B)$ for $B$ in $M_{E_{1} H}$. Finally, we define the linear isometry $W=\sum_{\alpha \in \mathfrak{l}} \phi\left(V_{\alpha}\right) W_{0} V_{\alpha}^{*}$; and if $\mathfrak{A}_{0}$ is any finite subset of $\mathfrak{A}$, then

$$
\begin{aligned}
& W\left(\sum_{\alpha \in \mathfrak{\Re}_{0}} E_{\alpha}\right) B\left(\sum_{\alpha \in \mathfrak{\Re}_{0}} E_{\alpha}\right) W^{-1} \\
& =\sum_{\alpha, \beta \in \mathfrak{A}_{0}} W\left(E_{\alpha} B E_{\beta}\right) W^{-1} \\
& \left.=\sum_{\alpha, \beta \in \mathfrak{F}_{0}} \phi\left(V_{\alpha}\right) W_{0} V_{\alpha}^{*} B V_{\beta} W_{0}^{-1} \phi\left(V_{\beta}^{*}\right) \quad \text { (since } W E_{\alpha}=\phi\left(V_{\alpha}\right) W_{0} V_{\alpha}^{*}\right) \\
& =\sum_{\alpha, \beta \in \mathfrak{A}_{0}} \phi\left(V_{\alpha}\right) \phi\left(V_{\alpha}^{*} B V_{\beta}\right) \phi\left(V_{\beta}^{*}\right) \text { (by properties of } W_{0} \text { ) } \\
& =\sum_{\alpha, \beta \in \mathfrak{H}_{0}} \phi\left(V_{\alpha} V_{\alpha}^{*} B V_{\beta} V_{\beta}^{*}\right)=\sum_{\alpha, \beta \in \mathfrak{R}_{0}} \phi\left(E_{\alpha} B E_{\beta}\right) \\
& =\sum_{\alpha, \beta \in \mathfrak{A}_{0}} \tilde{E}_{\alpha} \phi(B) \tilde{E}_{\beta} \\
& =\left(\sum_{\alpha \in \mathscr{A}_{0}} \tilde{E}_{\alpha}\right) \phi(B)\left(\sum_{\alpha \in \mathscr{\Re}_{0}} \tilde{E}_{\alpha}\right) .
\end{aligned}
$$

Again an obvious continuity argument yields finally that, for $B \in M$,

$$
W B W^{-1}=\phi(B),
$$

and the proof of Case 3 as well as that of the theorem is complete.

3. The coupling operator in arbitrary rings of operators. In [3] we introduced a coupling operator for substantial rings of operators. Utilizing the results of the earlier sections of this paper, we are now ready to define one for any ring of operators. Also we shall be able to extend many of the results of [3] concerning the standard topologies of operator rings. First we make

Definition 3.1. An arbitrary ring of operators can be expressed as the direct sum of a substantial part and a type III part. In the substantial part we have defined in Definition 1.6, page 483, of [3] a coupling operator; and in the type III part we have such an operator from Definition 1.3 of this paper. We now form the formal sum of these operators to give a formal operator which we shall call the coupling operator of our arbitrary ring.

If the substantial part of the coupling operator is essentially bounded, then 
we say that the coupling operator itself is essentially bounded. If our coupling operator is essentially finite and the ordinary operator part is bounded, then we say that the coupling operator itself is bounded.

Immediately we are able to prove

THEOREM 3. Let $M, \tilde{M}$ be rings of operators on Hilbert spaces $H, \widetilde{H}$ respectively. If $C$ and $\tilde{C}$ are the respective coupling operators and are essentially finite, and if $\phi$ is $a^{*}$-isomorphism of $M$ onto $\tilde{M}$ taking $C$ into $\tilde{C}$, then there exists a linear isometry $W$ mapping $H$ onto $\tilde{H}$ such that $\phi(A)=W A W^{-1}$ for $A \in M$. In particular, if $\phi$ is $a^{*}$-automorphism of $M$ leaving the center pointwise fixed, then there exists a unitary operator $W$ on $H$ such that $\phi(A)=W A W^{*}$ for each $A \in M$.

Proof. We split the ring $M$ into the direct sum of a substantial part and a type III part. By hypothesis, the coupling operators for these parts are taken over into the coupling operators for the corresponding split parts of $\tilde{M}$, the coupling operators for the substantial parts being essentially finite. Thus we can invoke Theorem 9, page 497 of [3] to get a linear isometry implementing $\phi$ on the substantial part. Finally, we can use Theorem 2 above to implement $\phi$ on the type III part with a linear isometry which can be pieced together with our other isometry to get one in the large. This proves the first part of our theorem.

In the case of a ${ }^{*}$-automorphism, the fact that $C$ is left fixed shows that we can use the first part of the proof of this theorem to obtain a linear isometry $W$ mapping $H$ onto itself and implementing $\phi$. But this means that $W$ is unitary and $W^{-1}=W^{*}$. Hence, for $A \in M$, we get $\phi(A)=W A W^{-1}$ $=W A W^{*}$, and the proof of this theorem is complete.

Lemma 3.1. Let $M$ be a ring of type III on a Hilbert space $H$. If

$$
C=\sum_{n, n^{\prime} \in \mathfrak{N}}\left(n / n^{\prime}\right) P_{n} P_{n^{\prime}}
$$

is the form of the coupling operator used in Definition 1.3 and if we apply the process outlined in Theorem 4, page 483 of [3] to the ring $M^{\prime}$ (with $m$ as our cardinal), then the rings $\tilde{M}, \tilde{M}^{\prime}$ obtained in this manner have the coupling operator $\tilde{C}=\sum_{n, n^{\prime} \in \mathfrak{N}}\left(n / m n^{\prime}\right) \tilde{P}_{n} \tilde{P}_{n^{\prime}}^{\prime}$.

Proof. Since it is clearly sufficient to prove that $\widetilde{P}_{n} \widetilde{P}_{n^{\prime}}^{\prime}$ has dimensions $n, n^{\prime}$ relative to the rings $\widetilde{M}, \widetilde{M}^{\prime}$ respectively, for arbitrary $n, n^{\prime}$ in $\Re$, let $Q=P_{n} P_{n^{\prime}}^{\prime}$ and $\widetilde{Q}=\widetilde{P}_{n} \widetilde{P}_{n^{\prime}}^{\prime}$. Then, using the notation of Theorem 4, page 483 of [3], we get a ${ }^{*}$-isomorphism $\phi$ of $M$ onto $\tilde{M}$ (our present $M$ takes the place of the $M^{\prime}$ of Theorem 4); and thus we see that our present Theorem 1 implies that $\widetilde{Q}=\phi(Q)$ has dimension $n$ relative to $\tilde{M}$.

Next, noticing that there is no loss in generality in assuming that $Q$ has u.d. $n^{\prime}$ relative to $M^{\prime}$ and replacing $M$ by $M Q$, we see that it is now sufficient 
to show that $I$ has u.d. $n^{\prime}$ relative to $\tilde{M}^{\prime}$. Further, using $\mathfrak{A}$ as our index set of cardinal $m$, the fact that each of the $\tilde{M}_{e_{\alpha}}^{\prime} \tilde{H}(\alpha \in \mathfrak{A})$ is unitarily equivalent to $M^{\prime}$ implies that each $e_{\alpha}$ has u.d. $n^{\prime}$ relative to $\tilde{M}_{e_{\alpha} \tilde{H}}^{\prime}$; and thus, for a fixed element $\alpha_{0} \in \mathfrak{A}$, there exists a collection $\left(\tilde{E}_{\alpha_{0}, \beta}^{\prime} \mid \beta \in \mathfrak{B}\right)$ of orthogonal, equivalent, cyclic projections in $\tilde{M}_{e_{\alpha} 0 \tilde{H}}^{\prime}$ such that $\sum_{\beta \in \mathscr{B}} \tilde{E}_{\alpha_{0}, \beta}=e_{\alpha_{0}}$. Also, since Theorem 1 implies that the $\tilde{E}_{\alpha_{0}, \beta}^{\prime}$ are cyclic relative to $\tilde{M}^{\prime}$ and since the projections $\left(e_{\alpha} \mid \alpha \in \mathfrak{A}\right)$ form an orthogonal, equivalent set, we can find a collection $\left(\tilde{E}_{\alpha, \beta}^{\prime} \mid \alpha \in \mathfrak{A}, \beta \in \mathfrak{B}\right)$ of orthogonal, equivalent, cyclic projections in $\tilde{M}^{\prime}$ such that for each $\alpha \in \mathfrak{A}, \sum_{\beta \in \mathcal{B}} \widetilde{E}_{\alpha, \beta}^{\prime}=e_{\alpha}$. It now follows easily from the equation $\sum_{\alpha \in \mathfrak{A}} e_{\alpha}=\tilde{I}$ that $\tilde{I}$ has, in $\tilde{M}^{\prime}$, a uniform dimension $N=$ (cardinality of the set $\mathfrak{A} \otimes \mathfrak{B})=m n^{\prime}$; and the proof of the present lemma is complete.

THEOREM 4. The strongest and $\sigma$-weak topologies are purely algebraic, that is, any ${ }^{*}$-isomorphism between rings of operators is bicontinuous in the strongest and $\sigma$-weak topologies.

Proof. Again, as in the proof of Theorem 3, we split our *-isomorphic rings into substantial and type III parts. On the substantial parts we quote Theorem 11, page 498 of [3] to show that on them the required topologies are purely algebraic. Thus, since addition is continuous, it is now sufficient to prove our theorem in the case of a type III ring.

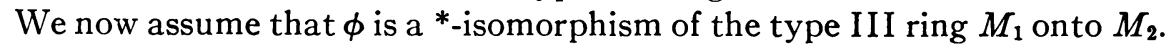
The proof now proceeds in the same manner as that of Theorem 11, page 498 of [3] with our present Theorem 3 and Lemma 3.1 replacing the Theorem 9 and Lemma 3.3 .1 of [3].

THEOREM 5. The notion of subring is purely algebraic, that is, if $\phi$ is a *-isomorphism of the ring $M$ onto the ring $\tilde{M}$ and if $N$ is a subring of $M$, then $\phi(N)$ is a subring of $\tilde{M}$.

Proof. The proof of this theorem is the same as that of Theorem 14, page 500 of $[3]$.

THEOREM 6. Let $C$ be the essentially bounded coupling operator for the rings $M, M^{\prime}$ on the Hilbert space $H . C$ is bounded if and only if the strongest and the strong, the $\sigma$-weak and the weak topologies are equivalent pairs of topologies.

Proof. First, let $C$ be bounded. As in the proof of Theorem 3, we split our ring $M$ into substantial and type III parts; and, since Theorem 12, page 499 of [3] takes care of the substantial part, we can now assume that $M$ is of type III.

Let $C=\sum_{n, n^{\prime} \in \mathfrak{N}}\left(n / n^{\prime}\right) P_{n} P_{n^{\prime}}^{\prime}$ and apply Lemma 3.1 to get rings $\tilde{M}, \tilde{M}^{\prime}$ with coupling operator $\widetilde{C}=\sum_{n, n^{\prime} \in \mathfrak{N}}\left(n / n^{\prime} \aleph_{0}\right) \widetilde{P}_{n} \tilde{P}_{n^{\prime}}^{\prime}=\sum_{n, n^{\prime} \in \mathfrak{N}}\left(n / n^{\prime}\right) \widetilde{P}_{n} \widetilde{P}_{n^{\prime}}^{\prime}$, if we assume that $m=\boldsymbol{\aleph}_{0}$. Thus, using the notation of Theorem 4 , page 483 of [3], we have $\widetilde{C}=\phi(C)$; and Theorem 2 gives us a linear isometry $W$ implementing $\phi$. 
Now, let $\left(x_{k} \mid k \in \Omega=\right.$ positive integers = index set used in the application of Theorem 4, page 483 of [3]) be a sequence of vectors in $H$ such that $\sum_{k \in \Re}\left\|x_{k}\right\|^{2}$ is finite; and, using the notation of [3], define $\tilde{x}_{k}=V_{k} x_{k}$ for $k \in \Re$. Clearly we can define a vector $\tilde{x}=\sum_{k \in \Omega} \tilde{x}_{k}$ in $\tilde{H}$; and, for $A \in M$, $\sum_{k \in \Re}\left(A V_{k}^{-1} \tilde{x}_{k}, \quad V_{k}^{-1} \tilde{x}_{k}\right)=\sum_{k \in \Omega}\left(A x_{k}, x_{k}\right)=(\phi(A) \tilde{x}, \tilde{x})=\left(W A W^{-1} \tilde{x}, \quad \tilde{x}\right)$ $=\left(A W^{-1} \tilde{x}, W^{-1} \tilde{x}\right)$. Thus we have proved that $\sum_{k \in \Re}\left(A x_{k}, x_{k}\right)=\left(A W^{-1} \tilde{x}\right.$, $\left.W^{-1} \tilde{x}\right)$ and it is easy to see that the strongest and the strong, the $\sigma$-weak and the weak are equivalent pairs of topologies.

Now suppose that the above pairs of topologies are equivalent. Then, by Theorem 13, page 499 of [3], the trace in the finite part of our ring $M$ is continuous in the strong topology; and, by Theorem 8, page 490 of [3], the coupling operator for the finite part must be bounded. But Definition 3.1 tells us that this means that our operator $C$ is bounded and we have completed the proof of this theorem.

We bring our paper to a close by indicating the proof of

THEOREM 7. Let $C, \tilde{C}$ be the essentially bounded coupling operators for the rings $M, M^{\prime}, \tilde{M}, \tilde{M}^{\prime}$, which act on the Hilbert spaces $H, \widetilde{H}$ respectively. Further, let $C_{0}$ and $\tilde{C}_{0}$ be the ordinary operator parts of the coupling operators, plus $\tilde{S}=$ L.U.B. $\left(\tilde{C}_{0}, \tilde{I}\right)$. Now, if $\phi$ is a ${ }^{*}$-isomorphism of $M$ onto $\tilde{M}$, then $\phi$ is strongly (weakly) continuous if and only if the operator $T=C_{0} \phi^{-1}\left(\tilde{S}^{-1}\right)$ is bounded.

Proof. It is obvious that our continuity condition is independent of the type III part of our ring $M$; and, since Theorems 4 and 6 yield strong (weak) continuity of $\phi$ on this part of $M$, our present theorem is proved there. But, on the substantial part, our theorem follows directly from Theorem A, page 503 of [3]; and our theorem is completely proved.

\section{REFERENCES}

1. J. Diximier, Les anneaux d'opérateurs de classe finie, Ann. Ecole Norm. vol. 66 (1949) pp. 209-261.

2. H. A. Dye, The Radon-Nikodym theorem for finite rings of operators, Trans. Amer. Math. Soc. vol. 72 (1952) pp. 243-280.

3. E. L. Griffin, Jr., Some contributions to the theory of rings of operators, Trans. Amer. Math. Soc. vol. 75 (1953) pp. 471-504.

4. Y. Misonou, Unitary equivalence of factors of type III, Proceedings of the Japan Academy vol. 29 pp. $482-485$.

5. F. J. Murray and J. von Neumann, On rings of operators, Ann. of Math. vol. 37 (1936) pp. 116-229.

6. J. von Neumann, On rings of operators. IV, Ann. of Math. vol. 44 (1943) pp. 716-808.

7. - On some algebraic properties of operator rings, Ann. of Math. vol. 44 (1943) pp. 709-715.

University OF Michigan, AnN Arbor, Mich. 